\title{
CLASSE EQUIVARIANTE DE
}

\author{
CHERN-SCHWARTZ-MACPHERSON
}

\author{
EQUIVARIANT CHERN-SCHWARTZ-MACPHERSON CLASS
}

\author{
AMANDA MONTEIRO \\ NIVALDO DE GÓES GRULHA JÚNIOR ${ }^{b}$
}

Resumo

Para uma variedade algébrica complexa singular existem várias definições de classes características possíveis. A classe de Chern-Schwartz-MacPherson é uma delas. R. MacPherson construiu a classe provando a existência de uma única transformação natural do grupo abeliano das funções construtíveis sobre $X$ para o grupo de homologia tal que, se $X$ é não-singular, então $C_{*}\left(1_{X}\right)$ coincide com a classe de Chern usual. Independentemente, M.-H. Schwartz introduziu classes de obstrução para a extensão de campos vetoriais radiais sobre $X$, e foi mostrado que essas definições são equivalentes, a partir de então esta classe tem sido chamada de classe de Chern-Schwartz-MacPherson.

Neste estudo, apresentamos uma $G$-versão da classe de Chern-SchwartzMacPherson para as $G$-variedades algébricas.

Palavras-chave: Variedade Singular, Classes Características, Teoria Equivariante.

\begin{abstract}
For a singular complex algebraic variety there are several possible characteristic class definitions. The Chern-Schwartz-MacPherson class is one of them. R. MacPherson constructed the class by proving the existence of a unique natural transformation from the abelian group of constructible functions over $X$ to the homology group so that if $X$ is nonsingular, then $C_{*}\left(1_{X}\right)$ coincide the usual Chern class. Independently, M.-H. Schwartz had introduced obstruction classes for the extension of stratified radial vector frames over $X$, and it was shown that these definitions are equivalent, henceforth this class has been called the Chern-Schwartz-MacPherson class.

In this study, we present a $G$-version of the Chern-Schwartz-MacPherson class for the algebraic $G$-varieties.
\end{abstract}

Keywords: Singular Variety, Characteristic Classes, Equivariant Theory.

MSC2010: $14 \mathrm{C} 17$

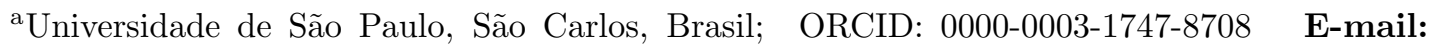
ammonteiro@usp.br

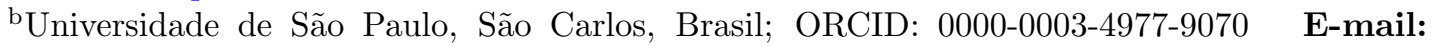
njunior@icmc.usp.br
} 


\section{Introdução}

Para uma variedade algébrica complexa singular existem várias definições de classes características possíveis. Tais definições neste contexto dependem da busca por substitutos para o fibrado tangente.

A classe de Chern-Schwartz-MacPherson é um exemplo destas classes. R. MacPherson construiu a classe para resolver a chamada conjectura de Grothendieck-Deligne: $\mathrm{Na}$ verdade, ele provou a existência de uma única transformação natural $C_{*}$ : $\mathcal{F}(X) \rightarrow H_{2 *}(X ; \mathbb{Z})$ do grupo abeliano $\mathcal{F}(X)$ das funções construtíveis sobre $X$ para o grupo de homologia (de dimensão par) tal que, se $X$ é não-singular, então $C_{*}\left(1_{X}\right)=c(T X) \frown[X]$, onde $1_{X}$ é a função característica, $1_{X}(x)=1$ para $x \in X$. De forma independente, M. H. Schwartz introduziu, em [7], classes de obstrução para a extensão de campos vetoriais radiais sobre $X$. Em [1] é mostrado que essas classes são as mesmas, via o isomorfismo de Alexander, a partir de então esta classe tem sido chamada de classe de Chern-Schwartz-MacPherson e é denotada frequentemente por $C^{S M}(X)$.

O objetivo destas notas é introduzir o leitor à $G$-versão da classe de ChernSchwartz-MacPherson para $G$-variedades algébricas $X$, apresentado em [5], detalhando os principais passos da construção formal da versão equivariante de $C_{*}$.

Para isso discutimos no contexto complexo, como em [4], usamos a cohomologia singular e a homologia de Borel-Moore, simplesmente denotada por $H_{*}(X)$, do espaço analítico (ou seja, localmente anelado) $\left(X, \mathcal{O}_{X}\right)$. Para nós, um esquema será sempre considerado separado, do tipo finito sobre um corpo $K$ de característica 0 , e uma variedade será um esquema integral.

Além da construção da $G$-versão da classe de Chern-Schwartz-MacPherson, também vamos abordar a teoria de polinômios de Thom nesta versão equivariante.

Para o desenvolvimento destas notas foram necessárias algumas ferramentas preliminares da Geometria Algébrica, de fibrados e de ações de grupos. Ao leitor interessado sugerimos consultar [3] e [2].

\section{Grupos Algébricos}

Nestas notas vamos considerar uma variedade sobre $K$ como sendo um $K$ esquema integral, do tipo finito e separado.

Definição 2.1. Um grupo algébrico $G$ (ou K-grupo) é definido como uma variedade sobre $K$ munida da topologia de Zariski, com uma estrutura de grupo tal que 
as aplicações

$$
\begin{aligned}
G \times G & \rightarrow G \\
(x, y) & \mapsto x . y
\end{aligned}
$$

$$
\begin{aligned}
G & \rightarrow G \\
x & \mapsto x^{-1}
\end{aligned}
$$

são morfismos de variedades.

Neste contexo, um homomorfismo de grupos algébricos $\alpha: G \rightarrow G^{\prime}$ é um morfismo de variedades sobre $K$ que também é homomorfismo de grupos. E um grupo algébrico $G$ é dito linear se a variedade algébrica $G$ é afim, ou seja, $G$ é um $K$-esquema afim.

Um exemplo clássico de grupo algébrico linear é qualquer subgrupo de $G L_{n}$ fechado com a topologia de Zariski (ver [8, p. 23]). Assim, dado um espaço vetorial $V$ de dimensão finita, podemos definir o grupo algébrico linear $G L(V)=G L_{\operatorname{dim} V}$.

Definição 2.2. Sejam $G$ um grupo algébrico e $X$ uma variedade sobre $K$. Dizemos que $X$ é uma $G$-variedade (ou $G$-espaço), se existe uma ação de $G$ em $X$ que é um morfismo de variedades. Mais precisamente, se existe um morfismo de variedades

$$
\begin{aligned}
\alpha: G \times X & \rightarrow X \\
(g, x) & \mapsto g x
\end{aligned}
$$

tal que $1_{G} x=x$ e $g(h x)=(g h) x$.

Dadas $X$ e $Y G$-variedades. Dizemos que um morfismo de $G$-variedades $\phi: X \rightarrow$ $Y$ é um $G$-morfismo (ou morfismo $G$-equivariante) se $\phi(g x)=g \phi(x), \forall g \in G, x \in$ $X$.

Definição 2.3. Seja $V$ um espaço vetorial de dimensão finita. Uma representação racional de $G$ em $V$ é um homomorfismo de grupos algébricos $r: G \rightarrow G L(V)$.

Mais geralmente, iremos nos referir ao espaço vetorial $n$-dimensional $V$, da definição anterior, como uma representação $n$-dimensional de $G$.

\section{$3 \quad$ Fibrados principais e associados}

Definição 3.1. Seja $\xi$ uma quíntupla $(E, B, \pi, F, G)$ composta de:

1. um espaço topológico E, chamado espaço total; 
2. um espaço topológico B, chamado espaço base;

3. uma aplicação contínua $\pi: E \rightarrow B$, chamada projeção;

4. um espaço topológico F chamado fibra típica;

5. um grupo topológico $G$ chamado grupo estrutural, com uma ação (à esquerda) livre

$$
\begin{aligned}
G \times F & \rightarrow F \\
(g, x) & \mapsto g x
\end{aligned}
$$

de $G$ na fibra típica $F$.

Dizemos que $\xi$ é um fibrado com grupo estrutural se satisfaz a condição de trivialidade local: existem uma cobertura aberta $\left\{U_{\alpha}\right\}_{\alpha \in A}$ de $B$ e uma família $\left\{\phi_{\alpha}\right\}_{\alpha \in A}$ de homeomorfismos

$$
\phi_{\alpha}: U_{\alpha} \times F \rightarrow \pi^{-1}\left(U_{\alpha}\right)
$$

$\operatorname{com} \pi \circ \phi_{\alpha}=\operatorname{pr}_{1}$ para todo $\alpha \in A$ (onde $p_{1}$ é a projeção na primeira coordenada), chamadas trivializações locais, tais que se a função $\phi_{\alpha, b}: F \rightarrow \pi^{-1}(b)$ é definida por

$$
\phi_{\alpha, b}(y)=(b, y)
$$

então para cada $\alpha, \beta \in A$ e cada $b \in U_{\alpha} \cap U_{\beta}$ o homeomorfismo

$$
\phi_{\alpha, b}^{-1} \circ \phi_{\beta, b}: F \rightarrow F
$$

coincide com a operação de um elemento do grupo $G$. E, ainda, para cada $\alpha, \beta \in A$ a função

$$
g_{\alpha \beta}: U_{\alpha} \cap U_{\beta} \rightarrow G
$$

definida por $g_{\alpha \beta}(b)=\phi_{\alpha, b}^{-1} \circ \phi_{\beta, b}$ é contínua.

Entre os fibrados com grupo estrutural, existe uma classe especial, os chamados fibrados principais:

Definição 3.2. Um fibrado $\xi=(E, B, \pi, F, G)$ é chamado fibrado principal (ou $G$-fibrado principal) se $F=G$ e $G$ age sobre si mesmo por translação à esquerda.

Um dos motivos para o uso do termo "fibrado principal" é o fato de que qualquer fibrado com grupo estrutural $G$ pode ser obtido a partir de um fibrado principal 
com grupo estrutural $G$ por um método direto e global: a construção do fibrado associado (ver mais detalhes em [9, Seção 9])).

Seja $P$ um fibrado principal sobre um espaço topológico $B$ com projeção $\pi: P \rightarrow$ $B$ e grupo estrutural $G$, e seja $Q$ um espaço topológico munido de uma ação de $G$

$$
\begin{aligned}
G \times Q & \rightarrow Q \\
(g, x) & \mapsto g x
\end{aligned}
$$

Considere o espaço produto $P \times Q$, munida da ação de $G$ à direita definida por

$$
\begin{array}{ccc}
(P \times Q) \times G & \rightarrow & P \times Q \\
((p, q), g) & \mapsto & (p, q) g=\left(p g, g^{-1} q\right)
\end{array}
$$

Denotaremos o espaço das órbitas desta ação por $P \times_{G} Q$, a projeção canônica de $P \times Q$ sobre $P \times_{G} Q$ por $\pi_{Q}$ e a classe de equivalência, ou órbita, de um par $(p, q)$ por $[p, q]$, assim, vale

$$
\left[p g, g^{-1} q\right]=[p, q] \text { ou }[p g, q]=[p, g q]
$$

para $p \in P, q \in Q, g \in G$.

Temos, então, $p r_{1} \circ \pi=\pi_{Q} \circ \bar{\pi}$, onde as aplicações $\bar{\pi}: P \times_{G} Q \rightarrow B$ e $\pi_{Q}$ : $P \times Q \rightarrow P \times{ }_{G} Q$ são dadas por $\bar{\pi}[p, q]=\pi(p)$ e $\pi_{Q}(p, q)=[p, q]$, para $p \in P, q \in Q$.

Nas condições anteriores, $\left(P \times_{G} Q, B, \bar{\pi}, Q, G\right)$ é chamado de fibrado associado ao fibrado principal $P$, mediante a ação dada de $G$ sobre sua fibra típica $Q$.

No nosso caso, estaremos interessados nos $G$-fibrados principais que tem como espaço total, espaço base e fibra típica as $G$-variedades. Nesta categoria, um morfismo entre $G$-fibrados principais $\left(P_{1}, B_{1}, \pi, G\right)$ e $\left(P_{2}, B_{2}, \pi^{\prime}, G\right)$ é qualquer aplicação $G$-equivariante $\phi: P_{1} \rightarrow P_{2}$.

Para qualquer morfismo de $G$-fibrados principais $\phi: P_{1} \rightarrow P_{2}$, existe uma aplicação $\widetilde{\phi}: B_{1} \rightarrow B_{2}$ tal que $\pi \circ \widetilde{\phi}=\pi^{\prime} \circ \phi$. A aplicação $\widetilde{\phi}: B_{1} \rightarrow B_{2}$ dada por $\widetilde{\phi}(b)=\pi^{\prime}(\phi(p))$, onde $b=\pi(p)$, está bem definida pois, dados $p, q \in \pi^{-1}(b)$, temos que $q=p g$ para algum $g \in G$ e, assim

$$
\pi^{\prime}(\phi(q))=\pi^{\prime}(\phi(p g))=\pi^{\prime}(\phi(p) g)=\pi^{\prime}(\phi(p))
$$

Definição 3.3. Dizemos que o fibrado principal $\xi=(E, \pi, B, G)$ é n-universal se $E$ é $(n-1)$-conexo, ou seja, $\pi_{i}(E)=0$, para $0 \leqslant i<n$. Dizemos, portanto, que $\xi$ é $\infty$-universal (ou universal) se $\pi_{i}(E)=0, \forall i$. 
Um espaço $B$ é um espaço classificante de $G$ se $B$ é espaço base de algum fibrado universal com grupo estrutural $G$. Quando nos referimos a um espaço classificante de $G$ qualquer, usa-se como notação habitual $B G$ para o espaço classificante e $E G$ para o espaço total do fibrado universal.

\section{Objetos na $G$-versão}

Seja $G$ um grupo algébrico linear redutivo de dimensão $g$. Tome $V$ uma representação $l$-dimensional de $G$ com um subconjunto $S$ fechado de Zariski $G$-invariante tal que $G$ age em $U:=V-S$ livremente.

Observação 4.1. É possível tomar $V$ e $S$ de forma que $U \rightarrow U / G$ se torne um $G$-fibrado principal sobre uma variedade quasi-projetiva e que a codimensão de $S$ seja suficientemente alta (ver [10, Remark 1.4]).

Seja $I(G)$ o conjunto de todos os abertos de Zariski $U=V-S$, onde $V$ é uma representação de $G$ e $S$ é um fechado de $V$ tais que todas as propriedades acima mencionadas são respeitadas. Agora, vamos colocar uma ordem parcial em $I(G)$ :

Definição 4.2. Sejam $U, U^{\prime} \in I(G)$. Dizemos que

$$
U(=V-S)<U^{\prime}\left(=V^{\prime}-S^{\prime}\right)
$$

se $\operatorname{codim}_{V} S<\operatorname{codim}_{V^{\prime}} S^{\prime}$ e se existe uma inclusão linear $G$-equivariante $i_{V, V^{\prime}}$ : $V \rightarrow V^{\prime}$ que leva $U$ em $U^{\prime}$.

Observação 4.3. $(I(G),<)$ é um conjunto direcionado, e portanto, podemos definir $I(G)^{*}$, a categoria dos indices $I(G)$.

Todos os $G$-fibrados principais $U \rightarrow U / G$ com as aplicações induzidas pelas inclusões formam um sistema direto (ou indutivo)

$$
\left\{U \rightarrow U / G,\left.i_{V, V^{\prime}}\right|_{U}\right\}_{U, U^{\prime} \in I(G)}
$$

que é a aproximação algébrica do fibrado universal $E G \rightarrow B G$. A descrição dessa aproximação, construída por B. Totaro, pode ser vista em [10].

Dado $U \in I(G)$ e uma $G$-variedade $X$, podemos construir

$$
X \times_{G} U \rightarrow U / G
$$


o fibrado associado a $U \rightarrow U / G$ como vimos anteriormente. A grosso modo, o fibrado universal $X \times{ }_{G} E G \rightarrow B G$ é aproximado por estes fibrados $X \times{ }_{G} U \rightarrow U / G$, $\operatorname{com} U \in I(G)$.

Definição 4.4. Sejam $U, U^{\prime} \in I(G)$, com $U<U^{\prime}, \iota_{U, U^{\prime}}: X \times_{G} U \rightarrow X \times_{G} U^{\prime} a$ inclusão natural e $r_{U, U^{\prime}}:=\iota_{U, U^{\prime}}^{*}: H^{*}\left(X \times{ }_{G} U^{\prime}\right) \rightarrow H^{*}\left(X \times{ }_{G} U\right)$ o homomorfismo induzido. Então, temos um sistema inverso (ou projetivo) $\left\{H^{*}\left(X \times_{G} U\right), r_{U, U^{\prime}}\right\}_{U, U^{\prime} \in I(G)}$. $O$ i-ésimo grupo de cohomologogia $G$-equivariante de $X$ é dado por

$$
H_{G}^{i}(X)=\lim _{\overleftarrow{I(G)}} H^{i}\left(X \times_{G} U\right)
$$

A soma formal é denotada por $H_{G}^{*}(X)=\prod H_{G}^{i}(X)=\lim _{\longleftarrow} H^{*}\left(X \times_{G} U\right)$. Também denotamos por $r_{U}: H_{G}^{*}(X) \rightarrow H^{*}\left(X \times{ }_{G} U\right)$ a projeção canônica para $U$.

Seja $\xi$ um fibrado vetorial $G$-equivariante $E \rightarrow X$, isto é, $E$ e $X$ são $G$-variedades e a projeção é $G$-equivariante de modo que a ação em $E$ preserva as fibras linearmente. Então, $\xi$ induz um fibrado vetorial $E \times{ }_{G} U \rightarrow X \times_{G} U$, denotado por $\xi_{U}$.

Definição 4.5. O limite inverso (ou projetivo) das classes de Chern c $\left(\xi_{U}\right)$ é a classe $G$-equivariante de Chern de $\xi$, que é denotada por $c^{G}(\xi) \in H_{G}^{*}(X)$.

Observação 4.6. Em particular, quando $X=\{p t\}$, um fibrado vetorial $G$-equivariante é $V \rightarrow\{p t\}$, sendo $V$ uma representação de $G$. A classe $G$-equivariante de Chern é denotada por $c^{G}(V) \in H_{G}^{*}(\{p t\})=H^{*}(B G)([5, p .5])$.

Vamos definir uma sub-ordem $<_{*}$ em $I(G)$ :

Definição 4.7. Dados quaisquer $U(=V-S)$ e $U^{\prime}\left(=V^{\prime}-S^{\prime}\right)$, dizemos que $U<_{*} U^{\prime}$ se existe uma representação $V_{1}$ de $G$ tal que $V \oplus V_{1}=V^{\prime}$ e $U \oplus V_{1}=U^{\prime}$.

Note que se $U_{1}<U_{2}$, então existe $U^{\prime}$ tal que $U^{\prime}<_{*} U_{1}$ e $U_{2}<_{*} U^{\prime}$ (por exemplo, $\left.U^{\prime}=V_{1} \oplus V_{2}-S_{1} \oplus S_{2}\right)$.

Observação 4.8. $\left(I(G),<_{*}\right)$ é um conjunto direcionado, e portanto, podemos definir $I(G)^{*}$ com relação a essa sub-ordem, a categoria dos índices $I(G)$.

Seja $G$-variedade complexa $X n$-dimensional. Para cada $U=V-S \operatorname{com} \operatorname{dim} V=$ $l$ e codim $S=s$, definimos a homologia truncada

$$
H_{\text {trunc }}\left(X \times_{G} U\right):=\bigoplus_{2(n-s)<i \leqslant 2 n} H_{i+2(l-g)}\left(X \times_{G} U\right) .
$$


Para cada par $U, U^{\prime} \in I(G)$ é possível definir um homomorfismo graduado dos grupos de homologia truncados, cujos graus são deslocados por $k=\operatorname{dim} U^{\prime}-\operatorname{dim} U$, denotado por

$$
\varphi_{U, U^{\prime}}: \bigoplus_{2(n-s)<i} H_{i+2(l-g)}\left(X \times_{G} U\right) \rightarrow \bigoplus_{2\left(n-s^{\prime}\right)<i} H_{i+2(l+k-g)}\left(X \times_{G} U\right),
$$

o que nos fornece um sistema direto (ou indutivo) com respeito ao conjunto direcionado $\left(I(G),<_{*}\right)$.

Definição 4.9. O i-ésimo grupo de homologia $G$-equivariante de $X$ é definido como o limite indutivo

$$
H_{i}^{G}(X)=\underset{I(G)}{\underset{I \rightarrow m}{\longrightarrow}} H_{i+2(l-g)}\left(X \times_{G} U\right) .
$$

Sendo $X$ uma $G$-variedade $n$-dimensional, estes grupos $H_{i}^{G}(X)$ são triviais para $i>2 n$ e possivelmente não trivial para qualquer $i$ negativo.

Temos então a soma direta

$$
H_{*}^{G}(X)=\oplus H_{i}^{G}(X)=\underset{I(G)}{\underset{\lim }{\longrightarrow}} H_{\text {trunc }}\left(X \times_{G} U\right) .
$$

Para cada $U$ a aplicação identificação é denotada por $\varphi_{U}: H_{t r u n c}\left(X \times_{G} U\right) \rightarrow$ $H_{*}^{G}(X)$.

Dado $U \in I(G)$ qualquer, a classe fundamental $\left[X \times_{G} U\right]$ tende a um único elemento de $H_{2 n}^{G}(X)$, denotado por $[X]_{G}$, chamado de classe fundamental $G$ equivariante de $X$.

Existe então um homomorfismo bem definido

$$
\begin{array}{cccc}
\frown[X]_{G}: H_{G}^{2 n-i}(X) & \rightarrow & H_{i}^{G}(X) \\
a & \mapsto \varphi_{U}\left(r_{U}(a) \frown\left[X \times_{G} U\right]\right)
\end{array}
$$

Observação 4.10. Se $X$ é não singular, então $\frown[X]_{G}$ é isomorfismo para cada $i$ chamado de Dualidade de Poincaré G-equivariante. Em particular, quando $X$ é um ponto,

$$
H_{-k}^{G}(p t) \simeq H_{G}^{k}(p t)=H^{k}(B G)
$$

Denotamos por Dual $_{G}$ o inverso da aplicação $\frown[X]_{G}$ (para cada $i$ ). A aplicação composta $r_{U} \circ D_{u a l} \circ \varphi_{U}$ coincide com o Dual de Poincaré de $X \times_{G} U$ na homologia truncada. 
Seja $X$ uma $G$-variedade. O subgrupo de $\mathcal{F}(X)$ que consiste das funções construtíveis $G$-invariantes é denotado por

$$
\mathcal{F}_{i n v}^{G}(X):=\{\alpha \in \mathcal{F}(X) \mid \alpha(g(x))=\alpha(x), x \in X, g \in G\}
$$

Dado qualquer $U<_{*} U^{\prime}\left(V^{\prime}=V \oplus V_{1}, U=V-S, U^{\prime}=V^{\prime}-S^{\prime}\right)$, seja $p r_{1}: V^{\prime} \rightarrow V$ a projeção na primeira coordenada. Então $p r_{1}$ induz um homomorfismo pullback

$$
\begin{array}{cc}
\phi_{U, U^{\prime}}:=\left(p r_{1}\right)^{*}: \mathcal{F}_{i n v}^{G}(X \times V) & \rightarrow \mathcal{F}_{i n v}^{G}\left(X \times V^{\prime}\right) \\
\alpha & \mapsto \alpha \circ\left(i d \times p r_{1}\right)
\end{array}
$$

(as vezes denotamos por $\phi_{V, V^{\prime}}$ ). Dessa forma, temos um outro sistema direto:

$$
\left\{\mathcal{F}_{i n v}^{G}(X \times V), \phi_{U, U^{\prime}}\right\}_{U, U^{\prime} \in I(G)}
$$

Definição 4.11. O grupo abeliano das funções G-equivariantes construtíveis $\mathcal{F}^{G}(X)$ é definido como limite indutivo

$$
\mathcal{F}^{G}(X):=\underset{I(G)}{\lim _{I(G v}} \mathcal{F}_{i n v}^{G}(X \times V)
$$

A aplicação identificação é denotada por $\phi_{U}: \mathcal{F}_{i n v}^{G}(X \times V) \rightarrow \mathcal{F}^{G}(X)$ (as vezes por $\left.\phi_{V}\right)$.

Para cada $U=V-S \in I(G)$ não vazio, a inclusão $U \subset V$, que vamos denotar por $j_{U}$, induz $j_{U}^{*}: \mathcal{F}_{i n v}^{G}(X \times V) \rightarrow \mathcal{F}_{\text {inv }}^{G}(X \times U)$.

Observação 4.12. Como $G$ atua livremente em $X \times U$, qualquer subesquema reduzido $G$-invariante $W$ de $X$ tem um $G$-fibrado principal $W \rightarrow W / G$. Assim, para $1_{W} \in \mathcal{F}_{\text {inv }}^{G}(X \times U)$ atribuimos $1_{W / G} \in \mathcal{F}_{\text {inv }}^{G}\left(X \times_{G} U\right)$, que na verdade cria um isomorfismo de grupos e deste modo, identificamos $\mathcal{F}_{\text {inv }}^{G}(X \times U)=\mathcal{F}_{\text {inv }}^{G}\left(X \times_{G} U\right)([5$, p. 10]).

Como $X \times_{G} U$ uma variedade algébrica complexa podemos considerar a transformação (comum) de MacPherson

$$
C_{*}: \mathcal{F}\left(X \times_{G} U\right) \rightarrow H_{*}\left(X \times_{G} U\right) .
$$

Vamos denotar por $T U_{G}$ o fibrado vetorial

$$
X \times_{G} T U\left(=X \times_{G}(U \oplus V)\right) \rightarrow X \times_{G} U
$$


e sua classe de Chern por $c\left(T U_{G}\right) \in H^{*}\left(X \times_{G} U\right)$. Ou seja, $c\left(T U_{G}\right):=r_{U}\left(c^{G}(V)\right)$, onde $r_{U}: H_{G}^{*}(X) \rightarrow H^{*}\left(X \times_{G} U\right)$ é a projeção canônica e $c^{G}(V)$ é a classe de Chern da representação $V$.

Combinando as aplicações acima, definimos

$$
T_{U, *}=c\left(T U_{G}\right)^{-1} \frown C_{*} \circ j_{U}^{*}: \mathcal{F}_{i n v}^{G}(X \times V) \rightarrow H_{*}\left(X \times_{G} U\right) .
$$

Definimos o homomorfismo limite como

$$
\begin{aligned}
C_{*}^{G}:=\lim _{\longrightarrow, *}: \mathcal{F}^{G}(X) & \rightarrow \quad H_{*}^{G}(X) \\
\phi_{U}\left(\alpha_{U}\right) & \mapsto \varphi_{U} \circ T_{U, *}\left(\alpha_{U}\right)
\end{aligned},
$$

onde $\phi_{U}: \mathcal{F}_{\text {inv }}^{G}(X \times V) \rightarrow \mathcal{F}^{G}(X)$ e $\varphi_{U}: H_{\text {trunc }}\left(X \times_{G} U\right) \rightarrow H_{*}^{G}(X)$ são aplicações de identificação.

O teorema a seguir garante que a aplicação $C_{*}^{G}$ é a transformação natural desejada, e que possui característica equivalentes a transformação natural (comum) de R. MacPherson. Para enunciarmos o teorema, precisamos relembrar que um grupo algébrico $G$ é redutivo se o maior subgrupo normal unipotente conexo suave de $G$ é trivial (mais detalhes em [8, 6.4.14]).

Teorema 4.13. Seja $G$ um grupo algébrico linear redutivo complexo. Para a categoria das $G$-variedades algébricas complexas e $G$-morfismos próprios, existe uma transformação natural de funtores covariantes

$$
C_{*}^{G}: \mathcal{F}^{G}(X) \rightarrow H_{*}^{G}(X)
$$

tal que se $X$ é não singular, então $C_{*}^{G}\left(1_{X}\right)=c^{G}(T X) \frown[X]_{G}$, onde $c^{G}(T X)$ é a classe G-equivariante de Chern total do fibrado tangente de X. A transformação natural $C_{*}^{G}$ é única em certo sentido.

A demonstração do resultado acima pode ser vista em [5, Theorem 1.1] e consiste em três passos. O primeiro passo é mostrar que $C_{G}^{*}$ é uma transformação natural (veja definição de transformação natural e exemplos em [6, Capítulo 8]), o segundo passo é demonstrar que se $X$ é não singular, então $C_{*}^{G}\left(1_{X}\right)=c^{G}(T X) \frown[X]_{G}$ o terceiro e último passo é demonstrar a unicidade de $C_{G}^{*}$ em certo sentido: supondo que para cada $U(=V-S)$ tenhamos um homomorfismo $D T_{U, *}: \mathcal{F}_{i n v}^{G}(X \times V) \rightarrow$ $H_{\text {trunc }}\left(X \times_{G} U\right)$ comutando com os homomorfismos estruturas $\left(\phi_{U, U^{\prime}}\right.$ e $\left.\varphi_{U, U^{\prime}}\right)$ tal que seu limite indutivo $D_{*}^{G}: \mathcal{F}^{G} \rightarrow H_{*}^{G}$ satisfaça as mesmas propriedades que $C_{G}^{*}$ e, então, como podemos tomar a codim $S$ suficientemente grande, podemos mostrar 
que $D_{G}^{*}=C_{G}^{*}$.

A partir deste teorema, de forma equivalente ao apresentado por R. MacPherson, pode-se definir então a versão equivariante da classe de Chern-Schwartz-MacPherson:

Definição 4.14. A classe G-equivariante de Chern-Schwartz-MacPherson de uma G-variedade $X$ é definida por

$$
C_{G}^{S M}(X):=C_{*}^{G}\left(1_{X}\right)
$$

\section{Polinômio de Thom}

Dada uma $G$-variedade $X$ podemos definir a aplicação

$$
\operatorname{Dual}_{G} \circ C_{*}^{G}: \mathcal{F}_{i n v}^{G}(X) \rightarrow H_{G}^{*}(X) .
$$

Para uma subvariedade $G$-invariante $W$ de codimensão $l$, o termo principal de $\operatorname{Dual}_{G} \circ C_{*}^{G}\left(1_{W}\right)$ é o dual de Poincaré $G$-equivariante de $[W]_{G}$ em $X$, que é chamado de polinômio de Thom de $W$ e é denotado por $t p(W) \in H_{G}^{2 l}(X)$.

Em particular, se $X$ é um $G$-espaço afim, sabemos que $H_{G}^{*}(X)=H^{*}(B G)$ e então $t p(W)$ é escrito como um polinômio de classes características $c_{i}$ de $G$-fibrados, que tem uma "universalidade" no seguinte sentido:

(Universalidade): Para qualquer fibrado $E \rightarrow M$ com fibra $X$ e grupo estrutural $G$ sobre um espaço base não singular $M$ de dimensão $m$, associamos um subfibrado $E_{W} \rightarrow M$ com fibra $W$. Dada uma seção genérica $s: M \rightarrow E$, definimos

$$
W(s):=s^{-1}\left(E_{W}\right)
$$

e chamamos $W(s)$ de conjunto singular do tipo $W$, que tem codimensão $l=$ $\operatorname{codim} W$. Seja $i: W(s) \rightarrow M$ a inclusão. Então, a classe fundamental do conjunto singular é expressa em $M$ por

$$
i_{*}[W(s)]=t p(W)(c(E)) \frown[M] \in H_{2(m-l)}(M)
$$

depois de substituir $c_{i}(E)$ por $c_{i}$ resultando em $t p(W) \in H^{2 l}(B G)$.

Definição 5.1. Para uma subvariedade invariantes $W$ em uma $G$-variedade $X$ não singular (denotamos a $G$-inclusão por $i: W \hookrightarrow X$ ), definimos a classe universal 
de Segre-SM de $W$ por

$$
s_{G}^{S M}(W, X):=c^{G}\left(\left.T X\right|_{W}\right)^{-1} \frown C_{*}^{G}\left(1_{W}\right) \in H_{*}^{G}(X),
$$

ou equivalentemente, $s_{G}^{S M}(W, X)=\varphi_{U}\left(s^{S M}\left(W \times_{G} U, X \times_{G} U\right)\right)$, onde $\varphi_{U}$ é a aplicação $H_{\text {trunc }}\left(W \times_{G} U\right) \rightarrow H_{*}^{G}(W)$. Seu dual G-equivariante em $X$ é denotado por

$$
t p^{S M}(W)=\operatorname{Dual}_{G} i_{*}^{G} s_{G}^{S M}(W, X) \in H_{G}^{*}(X) .
$$

Note que $t p^{S M}(W)$ é uma série de potências formal

$$
t p^{S M}(W)=\sum_{i=0}^{\infty} t p_{i}^{S M}(W) \in H_{G}^{*}(X)=\prod H_{G}^{i}(X)
$$

O teorema a seguir apresenta uma relação entre $t p^{S M}(W)$ e $t p(W)$. Para enunciarmos o teorema precisamos entender o que significa uma seção $s$ ser genérica com relação a $W$, para isso precisamos relembrar algumas definições:

Definição 5.2. Dizemos que $f: X \rightarrow Y$ é um morfismo de interseção completa local (morfismo i.c.l.) se $f$ é uma composição de um mergulho regular $i: X \rightarrow N$ e um morfismo suave $p: N \rightarrow Y$.

Definição 5.3. Seja $f: X \rightarrow Y$ um morfismo i.c.l. com fibrado normal virtual $\nu$. Dizemos que $f$ é genérico em relação a uma subvariedade $W$ de $Y$ se ele mantém que $C_{*} \circ f^{*}\left(1_{W}\right)=f^{* *} \circ C_{*}\left(1_{W}\right)$, ou seja,

$$
i_{*} C^{S M}\left(f^{-1}(W)\right)=c(\nu)^{-1} \frown f^{*} i_{*} C^{S M}(W)
$$

onde $i_{*}$ são as aplicações induzidas via inclusões.

Definição 5.4. Seja s uma seção de um fibrado $E \rightarrow M$ com fibra $X$ e grupo estrutural $G, W$ uma subvariedade $G$-invariante de $X$. Dizemos que s é genérico com relação a $W$ se o morfismo s é genérico com relação ao subfibrado associado $E_{W}$ com fibra $W$.

Com a definição acima podemos enunciar o teorema a seguir, nele assumimos que $M$ é uma variedade quasi-projetiva.

Teorema 5.5. Seja X um G-espaço afim e W uma subvariedade de X G-invariante de codimensão l. Então, 
1. $t p_{i}^{S M}(W)=0$, para $i<l$, e $t p_{l}^{S M}(W)$ coincide com o polinômio de Thom $t p(W)$ :

$$
t p^{S M}(W)=t p(W)+\text { termos mais altos; }
$$

2. (Universalidade) Para qualquer fibrado genérico $E \rightarrow M$ e qualquer seção $s$ genérica com relação a $W$, temos

$$
i_{*} C^{S M}(W(s))=t p^{S M}(W)(c(E)) \frown C^{S M}(M) \in H_{*}(M),
$$

onde $i_{*}$ denota a aplicação induzida pela inclusão.

A demonstração pode ser vista em [5, Theorem 7.5].

\section{Conclusão}

O estudo de classes características de variedades singulares é um tema atual e que tem sido amplamente utilizado em várias áreas da ciência. Assim como as classes características, as classes características equivariantes contribuem com informações topológicas relevantes dos objetos estudados e que são adaptáveis ao estudo dos espaços de órbitas.

Nessas notas apresentamos a construção de uma classe equivariante, dentre muitas possíveis, a de Chern-Schwartz-MacPherson, o que torna este trabalho um ponto de partida interessante para o estudo de outras classes como a de Mather, a de Fulton, dentre muitas outras e além outros resultados.

\section{Agradecimentos}

Gostaria de agradecer à FAPESP pelo apoio financeiro (Processo 2019/020688). Agradeco também pela leitura atenta, observações e sugestões do(a) parecerista que contribuíram grandemente para a melhoria destas notas. Trabalho desenvolvido durante doutorado no ICMC - USP, sob orientação do Prof. Dr. Nivaldo de Góes Grulha Júnior.

\section{Referências}

[1] BRASSELET, J.P.; Schwartz, M.H. Sur les classes de Chern d'une ensemble analytique complexe. Astérisque 82-83, p. 93-148, 1981. 
[2] GIBSON, C.G. Singular points of smooth mappings. Pitman Publishing, London, 1979.

[3] GÖRTZ, U.; Wedhorn, T. Algebraic Geometry I: Schemes with Examples and Exercises. Vieweg+teubner Verlag, 2010.

[4] MACPHERSON, R. Chern classes for singular algebraic varieties. Ann. of Math. 100, p. 421-432, 1974.

[5] OHMOTO, T. Equivariant Chern classes of singular algebraic varieties with group actions. Math. Proc. Cambridge Phil. Soc. 140, p. 115-134, 2006.

[6] RIBEIRO, M. F. S. Teoria das Categorias para Matemáticos; Uma breve introdução. SBM, Rio de Janeiro, 2020.

[7] SCHWARTZ, M.H. Classes caractéristiques définies par une stratification d'une variété analytique complere. CRAS 260, 3262-3264 et 3535-3537, 1965.

[8] SPRINGER, T.A. Linear Algebraic Groups. 2nd edition, Birkhäuser, 1998.

[9] STEEnROD, N. The Topology of Fibre Bundles. Princeton University Press, Princeton, 1999.

[10] TOTARO, B. The Chow Ring of a Classifying Space. Proc. Symposia in Pure Math., vol. 67, p. 249-281, 1999. 